Homology, Homotopy and Applications, vol.10(2), 2008, pp.273-286

\title{
CUP PRODUCTS IN HOPF CYCLIC COHOMOLOGY VIA CYCLIC MODULES
}

\author{
BAHRAM RANGIPOUR
}

\author{
(communicated by J. F. Jardine)
}

\begin{abstract}
We redefine the cup products in Hopf cyclic cohomology. These cup products were first defined by the author and M. Khalkhali via a relatively complicated method as a generalization of Connes' cup product for cyclic cohomology of algebras. In this paper we use the generalized Eilenberg-Zilber theorem and define the cup product using a bicocyclic module naturally associated to the cocyclic modules of the coalgebras and the algebras in question. In the last part of the paper we derive some formulas for the cup products.
\end{abstract}

\section{Introduction}

Hopf cyclic cohomology was invented by Connes and Moscovici as part of their fundamental work of computing the class of the index of the hypoelliptic signature operator [4]. They showed that the index cocycle is in the range of a characteristic map. The main ingredient of the characteristic map is an invariant trace. The characteristic map was generalized by Crainic [5] and Gorokhovsky [7] by using the fact that a cyclic cocycle on an algebra can be seen as a trace on the universal DG algebra of that algebra. This generalization is used in [3], where it is shown how a cup product is applicable in case the algebra under question possesses no invariant trace; as a replacement one takes advantage of an invariant cyclic cocycle to realize Hopf cyclic cocycles as cyclic cocycles on the algebra. Hopf cyclic cohomology was generalized to study Hopf-(co)module (co)algebras and coefficients (partially in [12] and completely in $[\mathbf{8}, \mathbf{9}])$. It was later generalized to encompass the category of bialgebra-(co)module (co)algebras [10]. In [9] it was conjectured that any characteristic map as above is a component of a cup product in Hopf cyclic cohomology. In [11] the author and M. Khalkhali proved the existence of the cup products via traces and cotraces over DG algebras and coalgebras.

We construct a cyclic cocycle on an algebra out of a Hopf cyclic cocycle on the algebra, and a Hopf cyclic cocycle on a coalgebra acting on the algebra. By simply tensoring the Hopf cocyclic modules of the algebra and coalgebra, we get a bicocyclic module. We first define a cyclic map from the diagonal of the bicocyclic module to

Received October 13, 2007, revised June 12, 2008; published on December 8, 2008. 2000 Mathematics Subject Classification: 16E40, 19D55, 16W30.

Key words and phrases: cup product, Hopf cyclic cohomology.

This article is available at http://intlpress.com/HHA/v10/n2/a14

Copyright (C) 2008, International Press. Permission to copy for private use granted. 
the cyclic module of the algebra. Then by using the Eilenberg-Zilber theorem we identify the cyclic cohomology of the diagonal mixed complex with that of the total mixed complex of our bicocyclic module. The fact that the equivariant property of cocycles yields that the produced cocycle will be well-defined on the convolution and crossed product algebra is nontrivial. There are at least eight kinds of cup products defined on Hopf cyclic cohomology but only two of them so far are applied in NCG; the reason could be their lack of classic and/or geometric counterparts. For the first product, one starts with Hopf cyclic cocycles over an algebra and a coalgebra with coefficients in a SAYD module. To define the cup product one needs the coalgebra to act on the algebra. The next step is to construct, via a twisting map, the cup product as a cocycle over the convolution algebra. But one knows that any cocycle over the convolution algebra is automatically a cocycle over the algebra. This cup product generalizes the characteristic map of Connes-Moscovici [4]. Ingredients for the second cup product are cyclic cocycles on a module algebra over a Hopf algebra and on a comodule algebra over the same Hopf algebra. Out of the two Hopf cyclic cocycles one produces a cyclic cocycle over the crossed product algebra. This cup product generalizes the ordinary cup product in cyclic cohomology of algebras as defined by Connes [1].

\section{Some preliminary definitions}

For the reader's convenience, we briefly recall the definition of Hopf cyclic cohomology of coalgebras and algebras under the symmetry of Hopf algebras and with coefficients in stable anti Yetter-Drinfeld (SAYD) modules $[\mathbf{4}, \mathbf{8}, \mathbf{9}]$. In this note, $\mathcal{H}$ is a Hopf algebra, and $\mu, \eta, \Delta, \varepsilon$, and $S$ are its product, unit, coproduct, counit and antipode, which is also assumed to be invertible. We use Sweedler's notation for the coproduct, i.e., $\Delta(h)=h_{(1)} \otimes h_{(2)}$. Let $C$ be an $\mathcal{H}$-module coalgebra, that is, a coalgebra endowed with an action, say from left, of $\mathcal{H}$ such that its comultiplication and counit are $\mathcal{H}$-linear, i.e.,

$$
\Delta(h c)=h_{(1)} c_{(1)} \otimes h_{(2)} c_{(2)}, \quad \varepsilon(h c)=\varepsilon(h) \varepsilon(c) .
$$

As the coefficients in this cohomology theory the notion of a SAYD module is defined in [8] and recalled as follows: It is said that a right module $M$, which is also a left comodule, is a right-left SAYD module over a Hopf algebra $\mathcal{H}$ if it satisfies the following conditions for any $h \in \mathcal{H}$ and $m \in M$ :

$$
\begin{gathered}
m_{<\overline{0}>} m_{<\overline{-1}>}=m, \\
(m h)_{<\overline{-1}>} \otimes(m h)_{<\overline{0}>}=S\left(h_{(3)}\right) m_{<\overline{-1}>} h_{(1)} \otimes m_{<\overline{0}>} h_{(2)},
\end{gathered}
$$

where the coaction of $\mathcal{H}$ is denoted by $\Delta_{M}(m)=m_{<\overline{-1}>} \otimes m_{<\overline{0}>}$.

Having the datum $(\mathcal{H}, C, M)$, one defines $[\mathbf{9}]$ the cocyclic module

$$
\left\{C_{\mathcal{H}}^{n}(C, M), \partial_{i}, \sigma_{j}, \tau\right\}_{n \geqslant 0}
$$

as follows:

$$
\mathcal{C}^{n}:=C_{\mathcal{H}}^{n}(C, M)=M \otimes_{\mathcal{H}} C^{\otimes n+1}, \quad n \geqslant 0,
$$


with the following cocyclic structure:

$$
\begin{aligned}
\partial_{i}: \mathcal{C}^{n} & \rightarrow \mathcal{C}^{n+1}, & & 0 \leqslant i \leqslant n+1 \\
\sigma_{j}: \mathcal{C}^{n} & \rightarrow \mathcal{C}^{n-1}, & & 0 \leqslant j \leqslant n-1, \\
\tau: \mathcal{C}^{n} & \rightarrow \mathcal{C}^{n}, & &
\end{aligned}
$$

defined explicitly as follows, where we abbreviate $\tilde{c}=c^{0} \otimes \cdots \otimes c^{n}$ :

$$
\begin{aligned}
\partial_{i}\left(m \otimes_{\mathcal{H}} \tilde{c}\right) & =m \otimes_{\mathcal{H}} c^{0} \otimes \cdots \otimes \Delta\left(c_{i}\right) \otimes \cdots \otimes c^{n}, \\
\partial_{n+1}\left(m \otimes_{\mathcal{H}} \tilde{c}\right) & =m_{<\overline{0}>} \otimes_{\mathcal{H}} c^{0}{ }_{(2)} \otimes c^{1} \otimes \cdots \otimes c^{n} \otimes m_{<-1>} c^{0}{ }_{(1)}, \\
\sigma_{i}\left(m \otimes_{\mathcal{H}} \tilde{c}\right) & =m \otimes_{\mathcal{H}} c^{0} \otimes \cdots \otimes \epsilon\left(c^{i+1}\right) \otimes \cdots \otimes c^{n}, \\
\tau\left(m \otimes_{\mathcal{H}} \tilde{c}\right) & =m_{<\overline{0}>} \otimes_{\mathcal{H}} c^{1} \otimes \cdots \otimes c^{n} \otimes m_{<-\overline{1}>} c^{0} .
\end{aligned}
$$

It is checked that the above defines a cocyclic module $[\mathbf{9}]$.

As the motivating example of the above theory, one recovers the cyclic complex of a Hopf algebra $\mathcal{H}$ endowed with a modular pair in involution $(\mathrm{MPI})(\delta, \sigma)$, which we recall from [4]. The character $\delta$ is an algebra map $\mathcal{H} \rightarrow \mathbb{C}$, and the group-like element $\sigma \in \mathcal{H}$ is a coalgebra map $\mathbb{C} \rightarrow \mathcal{H}$, i.e., $\sigma:=\sigma(1)$ satisfies $\Delta(\sigma)=\sigma \otimes \sigma$. The pair $(\delta, \sigma)$ is called MPI if $\delta(\sigma)=1$, and $\tilde{S}_{\delta}=\operatorname{Ad} \sigma$, where the twisted antipode $\tilde{S}_{\delta}$ is defined by

$$
\tilde{S}_{\delta}(h)=(\delta * S)(h)=\delta\left(h_{(1)}\right) S\left(h_{(2)}\right) .
$$

Similarly, an algebra which is an $\mathcal{H}$-module with $\mathcal{H}$-linear algebra structure is called an $\mathcal{H}$-module algebra. Let $A$ be an $\mathcal{H}$-module algebra. Then one endows $M \otimes A^{\otimes n+1}$ with the diagonal action of $\mathcal{H}$ and forms

$$
C_{\mathcal{H}}^{n}(A, M)=\operatorname{Hom}_{\mathcal{H}}\left(M \otimes A^{\otimes n+1}, \mathbb{C}\right)
$$

as the space of $\mathcal{H}$-linear maps. It is checked that the following defines a cocyclic module structure on $C^{n}(A, M)$ :

$$
\begin{array}{rlrl}
\left(\partial_{i} \varphi\right)(m \otimes \tilde{a}) & =\varphi\left(m \otimes a^{0} \otimes \cdots \otimes a^{i} a^{i+1} \otimes \cdots \otimes a^{n+1}\right), & & 0 \leqslant i<n, \\
\left(\partial_{n+1} \varphi\right)(m \otimes \tilde{a}) & =\varphi\left(m_{<\overline{0}>} \otimes\left(S^{-1}\left(m_{<\overline{-1}>}\right) a^{n+1}\right) a^{0} \otimes a^{1} \otimes \cdots \otimes a^{n}\right), & & \\
\left(\sigma_{i} \varphi\right)(m \otimes \tilde{a}) & =\varphi\left(m \otimes a^{0} \otimes \cdots \otimes a^{i} \otimes 1 \otimes \cdots \otimes a^{n-1}\right), & & 0 \leqslant i \leqslant n-1, \\
(\tau \varphi)(m \otimes \tilde{a}) & =\varphi\left(m_{<\overline{0}>} \otimes\left(S^{-1}\left(m_{<-\overline{1}>}\right) a^{n}\right) \otimes a^{0} \otimes \cdots \otimes a^{n-1}\right) . &
\end{array}
$$

The cyclic cohomology of this cocyclic module is denoted by $H C_{\mathcal{H}}^{*}(A, M)$.

An algebra is called an $\mathcal{H}$-comodule coalgebra if it is an $\mathcal{H}$-comodule and its coalgebra structure is $\mathcal{H}$ colinear. Similar to the other case, one defines ${ }^{\mathcal{H}} C^{n}(A, M)$ to be the space of all colinear maps from $A^{\otimes n+1}$ to $M$. One checks that the following defines a cocyclic module structure on ${ }^{\mathcal{H}} C^{n}(A, M)$ :

$$
\begin{aligned}
\left(\partial_{i} \varphi\right)(\tilde{a}) & =\varphi\left(a^{0} \otimes \cdots \otimes a^{i} a^{i+1} \otimes \cdots \otimes a^{n+1}\right), & & 0 \leqslant i<n, \\
\left(\partial_{n+1} \varphi\right)(\tilde{a}) & =\varphi\left(a^{n+1}{ }_{<0>} a^{0} \otimes a^{1} \cdots \otimes a^{n-1} \otimes a^{n}\right) a^{n+1}<-1> & & \\
\left(\sigma_{i} \varphi\right)(\tilde{a}) & =\varphi\left(a^{0} \otimes \cdots \otimes a^{i} \otimes 1 \otimes \cdots \otimes a^{n-1}\right), & & 0 \leqslant i \leqslant n-1, \\
(\tau \varphi)\left(a^{0} \otimes \cdots \otimes a^{n}\right) & =\varphi\left(a_{<0>}^{n} \otimes a^{0} \otimes \cdots \otimes a^{n-1} \otimes a^{n-1}\right) a_{<-1>}^{n} . & &
\end{aligned}
$$

The cyclic cohomology of this cocyclic module is denoted by ${ }^{\mathcal{H}} H C^{*}(A, M)$. 
Let $\left(C^{n}, \delta_{i}, \sigma_{i}, \tau_{n}\right)$ and $\left(C^{\prime n}, \delta_{i}, \sigma_{i}, \tau_{n}\right)$ be two cocyclic objects in the category of vector spaces. Their product is the cocyclic object $\left.\left(C \times C^{\prime}\right)^{n}, \delta_{i}, \sigma_{i}, \tau_{n}\right)$ with $\left(C \times C^{\prime}\right)^{n}=C^{n} \otimes C^{\prime n}$ and $\delta_{i}=\delta_{i} \otimes \delta_{i}, \sigma_{i}=\sigma_{i} \otimes \sigma_{i}$ and $\tau_{n}=\tau_{n} \otimes \tau_{n}$.

Their tensor product is the bicocyclic module $C \otimes C^{\prime}$ defined by $\left(C \otimes C^{\prime}\right)^{m, n}=$ $C^{m} \otimes C^{n}$ with horizontal and vertical structure borrowed from $C$ and $C^{\prime}$, respectively. The Eilenberg-Zilber theorem states that the cyclic cohomology of mixed complexes $C \times C^{\prime}$ and $\operatorname{Tot}\left(C \otimes C^{\prime}\right)$ are the same via the shuffle map [13].

\section{Module algebras paired with module coalgebras}

Let $\mathcal{H}$ be a Hopf algebra, $A$ be an $\mathcal{H}$-module algebra and $(\delta, \sigma)$ be a modular pair in involution on $\mathcal{H}$. Connes and Moscovici [4] showed that the following defines a map of cocyclic modules $\chi: \mathcal{H}_{(\delta, \sigma)}^{\natural} \rightarrow C^{*}(A)$ :

$$
\chi\left(h^{1} \otimes \cdots \otimes h^{n}\right)\left(a^{0} \otimes \cdots \otimes a^{n}\right)=\tau\left(a^{0} h^{1}\left(a^{1}\right) \cdots h^{n}\left(a^{n}\right)\right) .
$$

Here $\tau: A \rightarrow \mathbb{C}$ is a $\delta$-invariant $\sigma$-trace, i.e., for all $a, b$ and $h$

$$
\begin{aligned}
\tau(h a) & =\delta(h) \tau(a), \\
\tau(a b) & =\tau(b \sigma a) .
\end{aligned}
$$

The above map then induces the following characteristic map on the level of cohomology:

$$
\chi: H C_{(\delta, \sigma)}^{n}(\mathcal{H}) \rightarrow H C^{n}(A) .
$$

Hopf cyclic cohomology and SAYD (stable anti-Yetter-Drinfeld) modules generalize cyclic cohomology of Hopf algebras and MPI (modular pair in involution), respectively. Now a $\delta$-invariant $\sigma$-trace is exactly a closed cyclic cocycle in $C_{\mathcal{H}}^{0}\left(A,{ }^{\sigma} \mathbb{C}_{\delta}\right)$. These facts prompted us, in $[\mathbf{9}]$, to conjecture that there should exist a generalization of characteristic map as a pairing between Hopf cyclic cohomology of module algebras and module coalgebras:

$$
H C_{\mathcal{H}}^{n}(A, M) \otimes H C_{\mathcal{H}}^{m}(C, M) \rightarrow H C^{n+m}(A, M)
$$

where $M$ is a left-right SAYD module over $H$ and $C$ is an $\mathcal{H}$ module coalgebra acting on $A$ in the sense that there is a map

$$
C \otimes A \rightarrow A
$$

such that for any $h \in \mathcal{H}$, any $c \in C$ and any $a, b \in A$ one has

$$
\begin{aligned}
(h c) a & =h(c a), \\
c(a b) & =\left(c_{(1)} a\right)\left(c_{(2)} b\right), \\
c(1) & =\epsilon(c) 1 .
\end{aligned}
$$

Although there is a proof of the above conjecture in [11], we would like to give a more direct proof based on the theory of cyclic modules instead of traces on DG algebras. The advantage of this new view is its simplicity.

One constructs a convolution algebra $B=\operatorname{Hom}_{\mathcal{H}}(C, A)$, which is the algebra of all $\mathcal{H}$-linear maps from $A$ to $C$. The unit of this algebra is given by $\eta \circ \epsilon$, where $\eta: \mathbb{C} \rightarrow A$ 
is the unit of $A$. The multiplication of $f, q \in B$ is given by

$$
(f * g)(c)=f\left(c_{(1)}\right) g\left(c_{(2)}\right) .
$$

Now consider two cocyclic modules

$$
\left(C_{\mathcal{H}}^{*}(A, M), \delta_{i}, \sigma_{j}, t\right), \quad \text { and } \quad\left(C_{\mathcal{H}}^{*}(C, M), d_{i}, s_{j}, \tau\right)
$$

and let us make a new bicocyclic module by tensoring these over $\mathbb{C}$. The bicocyclic module has in bidegree $(p, q)$

$$
C^{p, q}:=\operatorname{Hom}_{\mathcal{H}}\left(M \otimes A^{\otimes p+1}, \mathbb{C}\right) \otimes\left(M \otimes_{\mathcal{H}} C^{\otimes q+1}\right),
$$

with horizontal structure $\vec{\partial}_{i}=d_{i} \otimes \mathrm{Id}, \vec{\sigma}_{j}=s_{j} \otimes \mathrm{Id}$, and $\vec{\tau}=t \otimes \mathrm{Id}$, and vertical structure $\uparrow \partial_{i}=\delta_{i} \otimes \mathrm{Id}, \uparrow \sigma_{j}=\sigma_{j} \otimes \mathrm{Id}$, and $\uparrow \tau=\tau \otimes \mathrm{Id}$. Here $\otimes:=\otimes$, which we use to separate $\operatorname{Hom}_{\mathcal{H}}\left(M \otimes A^{\otimes p+1}, \mathbb{C}\right)$ and $\left(M \otimes_{\mathcal{H}} C^{\otimes q+1}\right)$. Obviously,

$$
\left(C^{n, m}, \vec{\partial}, \vec{\sigma}, \vec{\tau}, \uparrow \partial, \uparrow \sigma, \uparrow \tau\right)
$$

defines a bicocyclic module.

Now let us define the map $\Psi_{c}: C^{n, n} \rightarrow \operatorname{Hom}\left(B^{\otimes n+1}, \mathbb{C}\right)$ by

$$
\Psi_{c}\left(\phi \otimes m \otimes c^{0} \otimes \cdots \otimes c^{n}\right)\left(f^{0} \otimes \cdots \otimes f^{n}\right)=\phi\left(m \otimes f^{0}\left(c^{0}\right) \otimes \cdots \otimes f^{n}\left(c^{n}\right)\right),
$$

which is obviously well-defined due to the facts that $f$ is $\mathcal{H}$-linear, $\phi$ is equivariant and (2) holds.

Proposition 3.1. The map $\Psi_{c}$ defines a cyclic map between the diagonal of $C^{*, *}$ and the cocyclic module $C^{*}(B)$.

Proof. We have to show that $\Psi$ commutes with the cyclic structures of the two sides. Indeed we just check it for the first face and cyclic operator and leave the rest to the reader. We have

$$
\begin{aligned}
\Psi_{c} & \left.\vec{\partial}_{0} \uparrow \partial_{0}\left(\phi \otimes m \otimes c^{0} \otimes \cdots \otimes c^{n}\right)\right)\left(f^{0} \otimes \cdots \otimes f^{n+1}\right) \\
& =\Psi_{c}\left(d_{0} \phi \otimes \delta_{0}\left(m \otimes c^{0} \otimes \cdots \otimes c^{n}\right)\right)\left(f^{0} \otimes \cdots \otimes f^{n+1}\right) \\
& =\phi\left(m \otimes f^{0}\left(c^{0}{ }_{(1)}\right) f^{1}\left(c^{0}{ }_{(2)}\right) \otimes f^{2}\left(c^{1}\right) \otimes \cdots \otimes f^{n+1}\left(c^{n}\right)\right. \\
& =\phi\left(m \otimes\left(f^{0} * f^{1}\right)\left(c^{0}\right) \otimes f^{2}\left(c^{1}\right) \otimes \cdots \otimes f^{n+1}\left(c^{n}\right)\right) \\
& =\left(d_{0} \Psi_{c}(\phi \otimes m \otimes \tilde{c})\right)\left(f^{0} \otimes \cdots \otimes f^{n+1}\right)
\end{aligned}
$$

and

$$
\begin{aligned}
\Psi_{c} & \left(\vec{\tau} \uparrow \tau\left(\phi \otimes m \otimes c^{0} \otimes \cdots \otimes c^{n}\right)\right)\left(a^{0} \otimes \cdots \otimes a^{n}\right) \\
& =\Psi_{c}\left(t \phi \otimes \tau\left(m \otimes c^{0} \otimes \cdots \otimes c^{n}\right)\right)\left(f^{0} \otimes \cdots \otimes f^{n}\right) \\
& \left.=t \phi\left(m_{<\overline{0}>} f^{0}\left(c^{1}\right) \otimes \cdots \otimes f^{n-1}\left(c^{n}\right)\right) \otimes m_{<-1>} f^{n}\left(c^{0}\right)\right) \\
& =\phi\left(m_{<\overline{0}>} \otimes S^{-1}\left(m_{<\overline{-1}>}\right) m_{<\overline{-2}>} f^{n}\left(c^{0}\right) \otimes f^{0}\left(c^{1}\right) \otimes \cdots \otimes f^{n-1}\left(c^{n}\right)\right) \\
& =\phi\left(m \otimes f^{n}\left(c^{0}\right) \otimes f^{0}\left(c^{1}\right) \otimes \cdots \otimes f^{n-1}\left(c^{n}\right)\right) \\
& =\left(t \Psi_{c}\left(\phi \otimes m \otimes c^{0} \otimes \cdots \otimes c^{n}\right)\right)\left(f^{0} \otimes \cdots \otimes f^{n}\right) .
\end{aligned}
$$


One can take advantage of properties (2), (3), and (4) to prove that there exists a natural unital algebra map $\natural: A \rightarrow \operatorname{Hom}_{\mathcal{H}}(A, C)$, explicitly defined by $\downarrow(a)(c)=c(a)$. As a result, one obtains a cyclic map $\downarrow: C^{*}(B, \mathbb{C}) \rightarrow C^{*}(A, \mathbb{C})$. One then composes $\downarrow$ with $\Psi_{c}$ to get a cyclic map

$$
\Psi=\natural \circ \Psi_{c}: C^{*}\left(\mathfrak{D}\left(C^{*, *}\right)\right) \rightarrow C^{*}(A, \mathbb{C}) .
$$

Let $\mathcal{K}$ be a sub Hopf algebra of $\mathcal{H}$. Although $A$ is an $\mathcal{H}$-module algebra, the coalgebra $\mathcal{C}=C(\mathcal{H}, \mathcal{K})=\mathcal{H} \otimes \mathcal{K} \mathbb{C}$ does not inherit this property from $\mathcal{H}$ since the action of $\mathcal{C}$ on $A$ is not well-defined. One cures this problem by letting $\mathcal{C}$ act on the invariant subalgebra of $A$ under the action of $\mathcal{K}$. Let

$$
A^{\mathcal{K}}=\{a \in A \mid k a=\varepsilon(k) a\} .
$$

One checks that the action of $\mathcal{C}$ on $A^{\mathcal{K}}$ is well-defined and satisfies (2), (3), and (4). One notes that it is not possible to write the map (5) for the case $A^{\mathcal{K}}$ and $\mathcal{C}$ because $A^{\mathcal{K}}$ is not an $\mathcal{H}$-module algebra. Instead one writes the invariant form of (5) as follows. Let us introduce

$$
C_{r}^{p, q}=C_{\mathcal{H}}^{p}(A, M) \otimes C_{\mathcal{H}}^{q}(\mathcal{H}, \mathcal{K} ; M)
$$

with its standard cyclic structure. Let us recall $C_{\mathcal{H}}^{q}(\mathcal{H}, \mathcal{K} ; M)$ from [2]. It is the cyclic module computing the Hopf cyclic cohomology of $\mathcal{H}$ relative to $\mathcal{K}$ with coefficients in $M$ and is defined as $H C_{\mathcal{H}}(\mathcal{C}, M)$, where $\mathcal{H}$ acts on $\mathcal{C}$ via $h \cdot(g \otimes 1)=h g \otimes 1$. Then one has a cyclic map $\Psi_{r}: \mathfrak{D}\left(C_{r}^{*, *}\right) \rightarrow C^{*}\left(A_{\mathcal{K}}\right)$ defined by

$$
\Psi_{r}\left(\phi \otimes m \otimes c^{0} \otimes c^{1} \otimes \cdots \otimes c^{n}\right)\left(a^{0} \otimes \cdots \otimes a^{n}\right)=\phi\left(m \otimes c^{0}\left(a^{0}\right) \otimes \cdots \otimes c^{n}\left(a^{n}\right)\right) .
$$

It is easily checked as in the absolute case that the above map defines a cyclic map. Note that it does not land in $C^{*}(A)$.

Corollary 3.2. The map $\Psi$ induces the following maps on cyclic cohomology groups:

$$
\begin{aligned}
\Psi: H C^{n}\left(\mathfrak{D}\left(C^{*, *}\right)\right) & \rightarrow H C^{n}(A), \\
\Psi_{c}: H C^{n}\left(\mathfrak{D}\left(C_{c}^{*, *}\right)\right) & \rightarrow H C^{n}\left(\operatorname{Hom}_{\mathcal{H}}(C, A)\right), \\
\Psi_{r}: H C^{n}\left(\mathfrak{D}\left(C_{r}^{*, *}\right)\right) & \rightarrow H C^{n}\left(A_{\mathcal{K}}\right) .
\end{aligned}
$$

We know [13], via the Eilenberg-Zilber theorem, that the total and diagonal mixed complexes are quasi isomorphic. Let us call this quasi isomorphism $\Phi$. One composes $\Psi, \Psi_{c}$, and $\Psi_{r}$ with $\Phi$ to obtain the following cup products in the level of cyclic cohomology:

$$
\begin{aligned}
\cup & =\Psi \circ \Phi: H C_{\mathcal{H}}^{p}(A, M) \otimes H C_{\mathcal{H}}^{q}(C, M) \rightarrow H C^{p+q}(A), \\
\cup & =\Psi_{c} \circ \Phi: H C_{\mathcal{H}}^{p}(A, M) \otimes H C_{\mathcal{H}}^{q}(C, M) \rightarrow H C^{p+q}\left(\operatorname{Hom}_{\mathcal{H}}(C, A)\right), \\
\cup & =\Psi_{r} \circ \Phi: H C_{\mathcal{H}}^{p}(A, M) \otimes H C_{\mathcal{H}}^{q}(\mathcal{H}, \mathcal{K} ; M) \rightarrow H C^{p+q}\left(A^{\mathcal{K}}\right) .
\end{aligned}
$$


Proposition 3.3. The above cup products are precisely given by the following formula in the level of Hochschild cohomology:

$$
\begin{gathered}
\cup: C_{\mathcal{H}}^{p}(A, M) \otimes C_{\mathcal{H}}^{q}(C, M) \rightarrow C^{p+q}(A), \\
\left(\phi \cup\left(m \otimes c^{0} \otimes \cdots \otimes c^{q}\right)\right)\left(a^{0} \otimes \cdots \otimes a^{p+q}\right)= \\
\phi\left(m_{<\overline{0}>} \otimes c^{0}{ }_{(p+1)}\left(a^{0}\right) c^{1}\left(a^{1}\right) \cdots c^{q}\left(a^{q}\right) \otimes m_{<-\bar{p}>} c_{(1)}^{0}\left(a^{q+1}\right) \otimes \cdots \otimes m_{<-\overline{1}>} c_{(p)}^{0}\left(a^{p+q}\right)\right) .
\end{gathered}
$$

Proof. The map $\Phi$ is a matrix map

$$
\Phi:=\left(\begin{array}{ccccc}
f^{0} & f^{1} & 0 & 0 & \cdots \\
0 & f^{0} & f^{1} & 0 & \cdots \\
\vdots & \vdots & \vdots & \vdots & \ddots
\end{array}\right) .
$$

The first component

$$
f^{0}: C^{p}(A, M) \otimes C^{q}(C, M) \rightarrow C^{p+q}(A, M) \otimes C^{p+q}(C, M)
$$

is defined by $\underbrace{d_{0} \cdots d_{0}}_{q \text { times }} \otimes d_{p+q} \cdots d_{q+1}$.

By composing $f^{0}$ and $\Psi$, one obtains the above formula. However, we prove that the formula defines a Hochschild cocycle by direct computation as we could not find a proof for it elsewhere. Indeed, one notes that if $\phi \in C_{\mathcal{H}}^{p}(A, M)$ and $x \in C_{\mathcal{H}}^{q}(C, m)$ are two Hochschild cocycles, then $\phi \otimes x$ is a Hochschild cocycle of degree $p+q$ in the total complex. We show that $b(\phi \cup x)=0$. Indeed, one uses the fact that $b(x)=0$ to see that

$$
\begin{aligned}
& \sum_{i=0}^{q}(-1)^{i} m \otimes c^{0} \otimes \cdots \otimes c^{i}{ }_{(1)} \otimes c_{(2)}^{i} \otimes \cdots \otimes c^{q}= \\
& (-1)^{q} m_{<\overline{0}>} \otimes c^{0}{ }_{(2)} \otimes c^{1} \otimes \cdots \otimes c^{q} \otimes m_{<-\overline{1}>} c_{(1)}^{0} .
\end{aligned}
$$

Applying the last face of $C_{\mathcal{H}}^{n}(C, M)$ repeatedly we get,

$$
\begin{aligned}
& m_{<\overline{0}>} \otimes c^{0}{ }_{(p+1)} \otimes c^{0}{ }_{(p+2)} \otimes c^{1} \otimes \cdots \otimes c^{q} \otimes m_{<\overline{-p-1}>} c^{0}{ }_{(1)} \otimes \cdots \otimes m_{<-\overline{1}>} c^{0}{ }_{(p)} \\
& +\sum_{i=1}^{q}(-1)^{i} m_{<\overline{0}>} \otimes c^{0}{ }_{(p+1)} \otimes c^{1} \otimes \cdots \otimes c^{i}{ }_{(1)} \otimes c^{i}{ }_{(2)} \otimes \cdots \otimes c^{q} \otimes \\
& m_{<-p-1>} c_{(1)}^{0} \otimes \cdots \otimes m_{<-1>} c_{(p)}^{0} \\
& =(-1)^{q} m_{<\overline{0}>} \otimes c_{(p+2)}^{0} \otimes c^{1} \otimes \cdots \otimes c^{q} \otimes m_{<-p-2>} c_{(1)}^{0} \otimes \cdots \otimes m_{<-\overline{1}>} c_{(p+1)}^{0} .
\end{aligned}
$$


On the other hand, one has

$$
\begin{aligned}
& b(\phi \cup x)\left(a^{0} \otimes \cdots \otimes a^{p+q+1}\right)= \\
& \phi\left(m_{<-1>} c_{(p+1)}^{0}\left(a^{0} a^{1}\right) c^{1}\left(a^{2}\right) \cdots c^{q}\left(a^{q+1}\right) \otimes\right. \\
& \left.m_{<-\overline{>}>} c^{0}{ }_{(1)}\left(a^{q+2}\right) \otimes \cdots \otimes m_{<-1>} c^{0}{ }_{(p)}\left(a^{p+q+1}\right)\right) \\
& +\sum_{i=1}^{q}(-1)^{i} \phi\left(m_{<-1>} c^{0}{ }_{(p+1)}\left(a^{0}\right) c^{1}\left(a^{1}\right) \cdots c^{i}\left(a^{i} a^{i+1}\right) c^{q}\left(a^{q+1}\right) \otimes\right. \\
& \left.m_{<-\overline{-p}>} c_{(1)}^{0}\left(a^{q+2}\right) \otimes \cdots \otimes m_{<-\overline{-1}>} c_{(p)}^{0}\left(a^{p+q+1}\right)\right) \\
& +\sum_{i=1}^{p}(-1)^{q+i} \phi\left(m_{<\overline{0}>} \otimes c^{0}{ }_{(p+1)}\left(a^{0}\right) c^{1}\left(a^{1}\right) \cdots c^{q}\left(a^{q}\right) \otimes\right. \\
& m_{<-\overline{-p}} c^{0}{ }_{(1)}\left(a^{q+2}\right) \otimes \cdots \otimes m_{<\overline{-p-i-1}>} c^{0}{ }_{(i)}\left(a^{q+i} a^{q+i+1}\right) \otimes \\
& \left.m_{<-p-i>} c^{0}{ }_{(i+1)}\left(a^{q+i+2}\right) \otimes \cdots \otimes \otimes_{<-1>} c^{0}{ }_{(p)}\left(a^{p+q+1}\right)\right) \\
& +(-1)^{p+q+1} \phi\left(m_{<-1>} c_{(p+1)}^{0}\left(a^{p+q+1} a^{0}\right) c^{1}\left(a^{2}\right) \cdots c^{q}\left(a^{q+1}\right) \otimes\right. \\
& \left.m_{<-p>} c_{(1)}^{0}\left(a^{q+2}\right) \otimes \cdots \otimes m_{<-1>} c_{(p)}^{0}\left(a^{p+q}\right)\right) .
\end{aligned}
$$

We use (2) and (6) to identify part of $b(\phi \cup x)\left(a^{0} \otimes \cdots \otimes a^{p+q+1}\right)$, which appears above in (7), with the following expression:

$$
\begin{gathered}
(-1)^{q} \phi\left(m_{<\overline{0}>} \otimes c_{(p+2)}^{0}\left(a^{0}\right) c^{1}\left(a^{1}\right) \cdots c^{q}\left(a^{q}\right) m_{<\overline{-p-2}>} c_{(1)}^{0}\left(a^{q+1}\right) \otimes\right. \\
\left.m_{<\overline{-p-1>}} c^{0}{ }_{(2)}\left(a^{q+2}\right) \otimes \cdots \otimes m_{<-1>} c^{0}{ }_{(p+1)}\left(a^{p+q+1}\right)\right),
\end{gathered}
$$

which yields that

$$
\begin{gathered}
b(\phi \cup x)\left(a^{0} \otimes \cdots \otimes a^{p+q+1}\right)= \\
(-1)^{q}(b \phi)\left(m_{<\overline{0}>} \otimes c^{0}{ }_{(p+2)}\left(a^{0}\right) c^{1}\left(a^{1}\right) \cdots c^{q}\left(a^{q}\right) \otimes m_{<\overline{-p-2}>} c^{0}{ }_{(1)}\left(a^{q+1}\right) \otimes\right. \\
m_{<\overline{-p-1}>} c^{0}{ }_{(2)}\left(a^{q+2}\right) \otimes \cdots \otimes m_{<\overline{-1}>} c^{0}{ }_{(p+1)}\left(a^{p+q+1}\right)=0 .
\end{gathered}
$$

Remark 3.4. For a modular pair in involution $(\delta, \sigma)$, it is shown that $\tau \in C^{0}\left(A,{ }^{\sigma} \mathbb{C}_{\delta}\right)$ if and only if $\tau$ is a $\delta$-invariant $\sigma$-trace on $A$ [9]. In addition, the above formula coincides with the characteristic map defined in [4]. As another example, let $C=\mathcal{H}:=$ $\mathcal{H}_{1}$, the Connes-Moscovici Hopf algebra [4], where it is shown that $1 \otimes \delta_{1} \in C_{\mathcal{H}}^{1}\left(\mathcal{H}, \mathbb{C}_{\delta}\right)$ is a cyclic cocycle. Here $\delta$ is the character on $\mathcal{H}$ defined in [4]. If $\phi \in C^{1}\left(A, \mathbb{C}_{\delta}\right)$ is a Hopf cyclic cocycle, then the above cup product formula $\phi \cup\left(1 \otimes \delta_{1}\right)$ coincides with the cocycle $g v$ constructed in [3], which is constructed via the cup product defined in [7]. These two coincidences hint to us that there should be another term in the general formula which makes the above a $b+B$ cocycle and disappears in special cases such as the above. By definition this term is the image of $f^{1}$.

\section{Module algebras paired with comodule algebras}

Let $\mathcal{H}$ be a Hopf algebra, $A$ a left $\mathcal{H}$-module algebra, $B$ a left $\mathcal{H}$-comodule algebra and $M$ be a right-left SAYD module over $\mathcal{H}$. One constructs a crossed product algebra whose underlying vector space is $A \otimes B$ with the $1 \rtimes 1$ as its unit and the following 
multiplication:

$$
(a \rtimes b)\left(a^{\prime} \rtimes b^{\prime}\right)=a b_{<-1>}\left(a^{\prime}\right) \rtimes b_{<0>} b^{\prime} .
$$

Now consider the two cocyclic modules

$$
\left(C_{\mathcal{H}}^{*}(A, M), \delta_{i}, \sigma_{j}, t\right), \quad \text { and } \quad\left({ }^{\mathcal{H}} C^{*}(B, M), d_{i}, s_{j}, \tau\right)
$$

introduced in [9] , and let us make a new bicocyclic module by tensoring these together. Its $(p, q)$ component $C^{p, q}$ is given by

$$
\operatorname{Hom}_{\mathcal{H}}\left(M \otimes A^{\otimes p+1}, \mathbb{C}\right) \otimes{ }^{\mathcal{H}} \operatorname{Hom}\left(B^{\otimes q+1}, M\right),
$$

with horizontal structure $\vec{\partial}_{i}=d_{i} \otimes \mathrm{Id}, \vec{\sigma}_{j}=s_{j} \otimes \mathrm{Id}$, and $\vec{\tau}=t \otimes \mathrm{Id}$, and vertical structure $\uparrow \partial_{i}=\delta_{i} \otimes \mathrm{Id}, \uparrow \sigma_{j}=\sigma_{j} \otimes \mathrm{Id}$, and $\uparrow \tau=\tau \otimes \mathrm{Id}$. Obviously,

$$
\left(C^{n, m}, \vec{\partial}, \vec{\sigma}, \vec{\tau}, \uparrow \partial, \uparrow \sigma, \uparrow \tau\right)
$$

defines a bicocyclic module. Now let us define a map

$$
\Psi: C^{n, n} \rightarrow \operatorname{Hom}\left((A \rtimes B)^{\otimes n+1}, \mathbb{C}\right)
$$

by

$$
\begin{aligned}
& \Psi(\phi \otimes \psi)\left(a^{0} \rtimes b^{0} \otimes \cdots \otimes a^{n} \rtimes b^{n}\right)= \\
& \phi\left(\psi\left(b_{<0>}^{0} \otimes \cdots \otimes b^{n}{ }_{<0>}\right) \otimes S^{-1}\left(b_{<1>}^{0} \cdots b_{<-1>}^{n}\right) a^{0} \otimes \cdots \otimes S^{-1}\left(b^{n}{ }_{<-n-1>}\right) a^{n}\right) .
\end{aligned}
$$

Proposition 4.1. The map $\Psi$ defines a cyclic map between the diagonal of $C^{*, *}$ and the cocyclic module $C^{*}(A \rtimes B)$.

Proof. We have to show that $\Psi$ commutes with the cyclic structures. We shall check it for the first face operator and the cyclic operator and leave the rest to the reader.

$$
\begin{aligned}
& \Psi\left(\vec{\partial}_{0} \uparrow \partial_{0}(\phi \otimes \psi)\right)\left(a^{0} \rtimes b^{0} \otimes \cdots \otimes a^{n+1} \rtimes b^{n+1}\right) \\
& \left.=\Psi\left(d_{0} \phi \otimes \delta_{0} \psi\right)\right)\left(a^{0} \rtimes b^{0} \otimes \cdots \otimes a^{n+1} \rtimes b^{n+1}\right) \\
& =\delta_{0} \phi\left(\delta_{0} \psi\left(b_{<0>}^{0} \otimes \cdots \otimes b^{n+1}{ }_{<0>}\right) \otimes\right. \\
& \left.S^{-1}\left(b_{<-1>}^{0} \cdots b_{<-1>}^{n+1}\right) a^{0} \otimes \cdots \otimes S^{-1}\left(b_{<-n-2>}^{n+1}\right) a^{n+1}\right) \\
& =\phi\left(\psi\left(b_{<0>}^{0} b_{<0>}^{1} \otimes \cdots \otimes b^{n+1}{ }_{<0>}\right) \otimes\right. \\
& S^{-1}\left(b_{<-1>}^{0} \cdots b_{<-1>}^{n+1}\right) a^{0} S^{-1}\left(b_{<-2>}^{1} \cdots b_{<-2>}^{n+1}\right) a^{1} \otimes \cdots \otimes \\
& \left.S^{-1}\left(b^{n+1}<-n-2>\right) a^{n+1}\right) \\
& =\phi\left(\psi\left(b_{<0>}^{0} b_{<0>}^{1} \otimes \cdots \otimes b^{n+1}{ }_{<0>}\right) \otimes\right. \\
& S^{-1}\left(b_{<-1>}^{0} \cdots b^{n+1}{ }_{<-1>}\right) a^{0} S^{-1}\left(b_{<-2>}^{1} \cdots b^{n+1}{ }_{<-2>}\right) a^{1} \otimes \cdots \otimes \\
& \left.S^{-1}\left(b_{<-n-2>}^{n+1}\right) a^{n+1}\right) \\
& =\phi\left(\psi\left(b_{<0>}^{0} b_{<0>}^{1} \otimes \cdots \otimes b^{n+1}{ }_{<0>}\right) \otimes\right. \\
& S^{-1}\left(b_{<-1>}^{0} b_{<-1>}^{1} \cdots b^{n+1}{ }_{<-1>}\right)\left(a^{0} b^{0}{ }_{<-2>} a^{1}\right) \otimes \cdots \otimes \\
& \left.S^{-1}\left(b^{n+1}<-n-1>\right) a^{n+1}\right) \\
& =\Psi(\phi \otimes \psi)\left(a^{0} b^{0}{ }_{<-1>} a^{1} \rtimes b^{0}{ }_{<0>} b^{1} \otimes a^{2} \rtimes b^{2} \otimes \cdots \otimes a^{n+1} \rtimes b^{n+1}\right) \\
& =d_{0} \Psi(\phi \otimes \psi)\left(a^{0} \rtimes b^{0} \otimes \cdots \otimes a^{n+1} \rtimes b^{n+1}\right) \text {. }
\end{aligned}
$$


Using the facts that $\phi$ is $\mathcal{H}$ equivariant, $\psi$ is $\mathcal{H}$ colinear and $M$ is SAYD one has:

$$
\begin{aligned}
& \Psi(\vec{\tau} \uparrow \tau(\phi \otimes \psi))\left(a^{0} \rtimes b^{0} \otimes \cdots \otimes a^{n} \rtimes b^{n}\right) \\
& =\Psi(t \phi \otimes \tau \psi)\left(a^{0} \rtimes b^{0} \otimes \cdots \otimes a^{n} \rtimes b^{n}\right) \\
& =t \phi\left(\tau \psi\left(b_{<0>}^{0} \otimes \cdots \otimes b_{<0>}^{n}\right) \otimes S^{-1}\left(b_{<1>}^{0} \cdots b_{<-1>}^{n}\right) a^{0} \otimes \cdots \otimes S^{-1}\left(b^{n}{ }_{<-n-1>}\right) a^{n}\right) \\
& =t \phi\left(\psi\left(b_{<0>}^{n} \otimes b_{<0>}^{0} \otimes \cdots \otimes b^{n-1}<0>\right) b_{<-1>}^{n} \otimes\right. \\
& \left.S^{-1}\left(b_{<1>}^{0} \cdots b^{n-1}{ }_{<-1>} b_{<-2>}^{n}\right) a^{0} \otimes \cdots \otimes S^{-1}\left(b_{<-n-2>}^{n}\right) a^{n}\right) \\
& =\phi\left(\left[\psi\left(b^{n}{ }_{<0>} \otimes b^{0}{ }_{<0>} \otimes \cdots \otimes b^{n-1}{ }_{<0>}\right) b^{n}{ }_{<-1>}\right]_{<0>} \otimes\right. \\
& S^{-1}\left(\left[\psi\left(b^{n}{ }_{<0>} \otimes b_{<0>}^{0} \otimes \cdots \otimes b^{n-1}{ }_{<0>}\right) b^{n}{ }_{<-1>}\right]_{<-1>}\left(S^{-1}\left(b^{n}{ }_{<-n-2>}\right) a^{n}\right) \otimes\right. \\
& \left.S^{-1}\left(b_{<1>}^{0} \cdots b_{<-1>}^{n-1} b_{<-2>}^{n}\right) a^{0} \otimes \cdots \otimes S^{-1}\left(b^{n-1}{ }_{<-n+1>} b_{<-n-1>}^{n}\right) a^{n-1}\right) \\
& =\phi\left(\psi\left(b_{<0>}^{n} \otimes b_{<0>}^{0} \otimes \cdots \otimes b^{n-1}{ }_{<0>}\right) b^{n}{ }_{<-3>} \otimes\right. \\
& \left.S^{-1}\left(S\left(b_{<-2>}^{n}\right) b^{n}{ }_{<-1>} b_{<-1>}^{0} \cdots b^{n-1}{ }_{<-1>}\right) b_{<-4>}^{n}\right)\left(S^{-1}\left(b^{n}{ }_{<-n-2>}\right) a^{n}\right) \otimes \\
& \left.S^{-1}\left(b_{<1>}^{0} \ldots b^{n-1}<_{<-1>} b_{<-5>}^{n}\right) a^{0} \otimes \cdots \otimes S^{-1}\left(b^{n-1}{ }_{<-n+1>} b_{<-n-4>}^{n}\right) a^{n-1}\right) \\
& =\phi\left(\psi\left(b^{n}{ }_{<0>} \otimes b^{0}{ }_{<0>} \otimes \cdots \otimes b^{n-1}{ }_{<0>}\right) b^{n}{ }_{<-1>} \otimes\right. \\
& \left.S^{-1}\left(b_{<-1>}^{0} \cdots b_{<-1>}^{n-1}\right) b_{<-2>}^{n}\right)\left(S^{-1}\left(b_{<-n-2>}^{n}\right) a^{n}\right) \otimes \\
& \left.S^{-1}\left(b_{<1>}^{0} \cdots b^{n-1}{ }_{<-1>} b_{<-3>}^{n}\right) a^{0} \otimes \cdots \otimes S^{-1}\left(b^{n-1}{ }_{<-n+1>} b_{<-n-2>}^{n}\right) a^{n-1}\right) \\
& =\phi\left(\psi\left(b_{<0>}^{n} \otimes b_{<0>}^{0} \otimes \cdots \otimes b^{n-1}{ }_{<0>}\right) \otimes S^{-1}\left(b_{<-1>}^{n} b_{<-1>}^{0} \cdots b^{n-1}{ }_{<-1>}\right) a^{n}\right) \otimes
\end{aligned}
$$

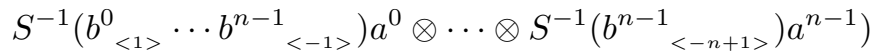

$$
\begin{aligned}
& =\Psi(\phi \otimes \psi)\left(a^{n} \rtimes b^{n} \otimes a^{0} \rtimes b^{0} \otimes \cdots \otimes a^{n-1} \rtimes b^{n-1}\right) \\
& =t \Psi(\phi \otimes \psi)\left(a^{0} \rtimes b^{0} \otimes \cdots \otimes a^{n} \rtimes b^{n}\right) .
\end{aligned}
$$

Corollary 4.2. The map $\Psi$ defined in (8) induces a map on cyclic cohomology:

$$
\Psi: H C^{n}\left(\mathfrak{D}\left(C^{*, *}\right)\right) \rightarrow H C^{n}(A \rtimes B) .
$$

Now by composing $\Psi$ with the corresponding $A W$ map one proves the existence of the following map:

$$
\cup=\Psi \circ \Phi: H C_{H}^{p}(A, M) \otimes{ }^{\mathcal{H}} H C^{q}(B, M) \rightarrow H C^{p+q}(A \rtimes B) .
$$

One uses the formula for the $A W$ map [13] to find the following expression for the above cup product.

Proposition 4.3. The above cup product has the following formula at the level of Hochschild cohomology.

$$
\begin{aligned}
& \phi \cup \psi\left(a^{0} \rtimes b^{0} \otimes \cdots \otimes a^{p+q} \rtimes b^{p+q}\right)= \\
& \phi\left(\psi\left(b^{q+1}{ }_{<0>} \cdots b^{p+q}{ }_{<0>} b^{0}{ }_{<0>} \otimes b^{1}{ }_{<0>} \otimes \cdots \otimes b^{q}{ }_{<0>}\right)_{<-1>} \otimes\right. \\
& S^{-1}\left(b_{<-1>}^{0} \cdots b^{q}{ }_{<-1>}\right) a^{0} \cdots S^{-1}\left(b_{<-q-1>}^{q}\right) a^{q} \otimes a^{q+1} \otimes \\
& \left.b^{q+1}{ }_{<-p-1>} a^{q+2} \otimes \cdots \otimes b^{q+1}<-1>\cdots b^{p+q-1}{ }_{<-1>} a^{p+q}\right) .
\end{aligned}
$$

Proof. The proof is similar to that of Proposition 3.3. 
Example 4.4. Let $G$ be a discrete group acting by unital automorphisms on an algebra $A$ and let $k$ be a field of characteristic zero. In [12], the Hopf cyclic cohomology groups of the Hopf algebra $H=k G$ were computed in terms of group cohomology with trivial coefficients:

$$
{ }^{k G} H C^{p}(k G, \mathbb{C})=\bigoplus_{i \geqslant 0} H^{p-2 i}(G, \mathbb{C}) .
$$

By considering the trivial SAYD $\mathbb{C}$, the cohomology groups $H C_{\mathbb{C} G}^{q}(A, \mathbb{C})$ are by definition the cohomology of the subcomplex of invariant cyclic cochains on $A$ :

$$
\varphi\left(g a_{0}, g a_{1}, \cdots, g a_{n}\right)=\varphi\left(a_{0}, a_{1}, \cdots, a_{n}\right),
$$

for all $g \in G$ and $a_{i} \in A$.

We denote this cohomology theory by $H C_{G}^{q}(A)$; thus we have a pairing

$$
H^{p}(G) \otimes H C_{G}^{q}(A) \longrightarrow H C^{p+q}(A \rtimes G) .
$$

\section{Cup product via traces}

In this section we derive some formulas for the cup products defined in [11].

Let us briefly recall it here. Let $A$ be a left $\mathcal{H}$ module algebra, $B$ a left $\mathcal{H}$-comodule algebra and $M$ a SAYD module on $\mathcal{H}$. Also let $\Omega A$ be a DG $\mathcal{H}$-module algebra over $A$ and $\Gamma B$ be a DG $\mathcal{H}$-comodule algebra over $B$. We recall that a closed $M$-trace on $\Omega A$ is a linear map $\int: M \otimes \Omega A \rightarrow \mathbb{C}$ such that

$$
\begin{gathered}
\int\left(h_{(1)} m \otimes h_{(2)} \omega\right)=\epsilon(h) \int(m \otimes \omega), \\
\int(m \otimes d \omega)=0, \\
\int\left(m \otimes \omega^{1} \otimes \omega^{2}\right)=(-1)^{\operatorname{deg}\left(\omega^{1}\right) \operatorname{deg}\left(\omega^{2}\right)} \int\left(m_{<\overline{0}>} \otimes S^{-1}\left(m_{<-\overline{-1}>}\right) \omega^{2} \omega^{1}\right) .
\end{gathered}
$$

Similarly, a closed $M$-trace on $\Gamma B$ is defined as a linear map $\int: \Gamma B \rightarrow M$ such that

$$
\begin{gathered}
\left(\int \gamma\right)_{<-1>} \otimes\left(\int \gamma\right)_{<\overline{0}>}=\gamma_{<-1>} \otimes \int\left(\gamma_{<0>}\right), \\
\int(d \gamma)=0, \\
\int\left(\gamma^{1} \gamma^{2}\right)=\int\left(\gamma_{<0>}^{2} \gamma^{1}\right) \gamma_{<-1>}^{2} .
\end{gathered}
$$

One identifies closed cyclic cocycles $\phi \in C_{\mathcal{H}}^{p}(A, M)$ and $\psi \in{ }^{\mathcal{H}} H C^{q}(B, M)$ with closed $M$-traces on $\Omega(A)$ and $\Gamma(B)$, the universal $\mathcal{H}$-module DG algebra and $\mathcal{H}$-comodule algebra respectively, as follows:

$$
\begin{gathered}
\int_{\phi} m \otimes a^{0} d a^{1} \cdots d a^{n} i=\phi\left(m \otimes a^{0} \otimes \cdots \otimes a^{p}\right), \\
\int_{\psi}\left(b^{0} d b^{1} \cdots d b^{n}\right)=\psi\left(b^{0} \otimes \cdots \otimes b^{q}\right) .
\end{gathered}
$$

Then one forms a DG algebra over $A \rtimes B$ as the crossed product of $\Omega(A)$ and 
$\Gamma(B)$, which we denote by $\Omega(A) \rtimes \Gamma(B)$. For any two closed $M$-traces $\int_{1}$ and $\int_{2}$ on $\Omega(A)$ and $\Gamma(B)$, one defines $[\mathbf{1 1}]$ the closed trace $\int_{1} \cup \int_{2}$ on $\Omega(A) \rtimes \Gamma(B)$ by

$$
\left(\int_{1} \cup \int_{2}\right)(\omega \rtimes \eta)=\int_{1}\left(\int_{2}(\omega) \otimes \eta\right) ;
$$

hence the cup product of two cyclic cocycles is defined by

$$
\begin{aligned}
(\phi \cup \psi)\left(a^{0} \rtimes b^{0} \otimes a^{1} \rtimes b^{1} \otimes\right. & \left.\cdots \otimes a^{p+q} \rtimes b^{p+q}\right)= \\
& \left(\int_{\phi} \cup \int_{\psi}\right)\left(a^{0} \rtimes b^{0} d\left(a^{1} \rtimes b^{1}\right) \cdots d\left(a^{p+q} \rtimes b^{p+q}\right)\right) .
\end{aligned}
$$

Now we want to derive a formula for the above cup product. To this end we need to know the $(p, q)$ component of the form

$$
\theta^{n}=a^{0} \rtimes b^{0} d\left(a^{1} \rtimes b^{1}\right) \cdots d\left(a^{n} \rtimes b^{n}\right) .
$$

For a permutation $\sigma \in \operatorname{Sh}(q, p)$, we use $\bar{\sigma}(i)=\sigma(i)-1$, and $\hat{\sigma}(i)=\sigma(i)+1$, and define the following $p+q$ form

$$
\begin{gathered}
\theta_{\sigma}^{n}=a^{0}\left(b^{0} \cdots b^{n-1}\right)_{<-n>} a^{1} \cdots\left(b^{\bar{\sigma}(q+1)-1} \cdots b^{n-1}\right)_{<-n+\sigma(q+1)-2>} a^{\bar{\sigma}(q+1)} \\
d\left(\left(b^{\bar{\sigma}(q+1)} \cdots b^{n-1}\right)_{<-n+\bar{\sigma}(q+1)>} a^{\sigma(q+1)} \cdots\left(b^{\bar{\sigma}(q+2)-1} \cdots b^{n-1}\right)_{<-n+\sigma(q+2)-2>} a^{\bar{\sigma}(q+2)}\right) \\
\cdots d\left(\left(b^{\bar{\sigma}(n)} \cdots b^{n-1}\right)_{<-n+\bar{\sigma}(n)>} a^{\sigma(n)} \cdots b^{n-1}{ }_{<-1>} a^{n}\right) \rtimes \\
b_{<0>}^{0} \cdots b_{<0>}^{\bar{\sigma}(1)} d\left(b_{<0>}^{\sigma(1)} \cdots b_{<0>}^{\bar{\sigma}(2)}\right) \cdots d\left(b_{<0>}^{\sigma(q)} \cdots b_{<0>}^{n-1} b^{n}\right) .
\end{gathered}
$$

Lemma 5.1. The $(q, p)$ th component of the above form $\theta^{p+q}$ is given by the following formula:

$$
\sum_{\sigma \in \operatorname{Sh}(q, p)}(-1)^{\sigma} \theta_{\sigma}^{p+q}
$$

Proof. We prove it by induction. Obviously it is true for $(p, q)=(0,0)$. Assume that the lemma is true for all $(p, q)$ such that $p+q=n$ We prove it for all $(p, q)$ that $p+q=n+1$.

The $(p, q)$ th component of

$$
a^{0} \rtimes b^{0} d\left(a^{1} \rtimes b^{1}\right) \cdots d\left(a^{p+q} \rtimes b^{p+q}\right)
$$

is

$$
\theta\left(d a^{p+q} \rtimes b^{p+q}\right)+\theta^{\prime}\left(a^{p+q} \rtimes d b^{p+q}\right),
$$

where $\theta$ and $\theta^{\prime}$ are the $(p-1, q)$ th and $(p, q-1)$ th components of

$$
a^{0} \rtimes b^{0} d\left(a^{1} \rtimes b^{1}\right) \cdots d\left(a^{p+q-1} \rtimes b^{p+q-1}\right),
$$


respectively. Now let $\mu \in \operatorname{Sh}(q, p-1)$. One observes that

$$
\begin{aligned}
\sum_{\sigma \in \operatorname{Sh}(q-1, p)} & (-1)^{\sigma} \theta_{\sigma}^{p+q-1}\left(a^{p+q} \rtimes d b^{p+q}\right)+\sum_{\sigma \in \operatorname{Sh}(q, p-1)}(-1)^{\sigma} \theta_{\sigma}^{p+q-1}\left(d a^{p+q} \rtimes b^{p+q}\right) \\
& =\sum_{\sigma \in \operatorname{Sh}(q, p)}(-1)^{\sigma} \theta_{\sigma}^{p+q}+\sum_{\sigma \in \operatorname{Sh}(q, p)} \sum_{\sigma(p+q)=p+q}(-1)^{\sigma} \theta_{\sigma}^{p+q} \\
& =\sum_{\sigma \in \operatorname{Sh}(q, p)}(-1)^{\sigma} \theta_{\sigma}^{p+q} .
\end{aligned}
$$

As a result one has the following formula for a cup product via traces:

Proposition 5.2. Let $\phi \in C_{\mathcal{H}}^{p}(A, M)$ and $\psi \in{ }^{\mathcal{H}} C^{q}(B, M)$, respectively, be two Hopf cyclic cocycles on $A$ and $B$ with coefficients in a SAYD module $M$. Then $\phi \cup \psi \in$ $C^{p+q}(A \rtimes B)$ is a cyclic cocycle, which is given precisely by the following formula:

$$
\begin{gathered}
(\phi \cup \psi)\left(a^{0} \rtimes b^{0} \otimes a^{1} \rtimes b^{1} \otimes \cdots \otimes a^{p+q} \rtimes b^{p+q}\right)= \\
\sum_{\sigma \in \operatorname{Sh}(q, p)}(-1)^{\sigma} \partial_{\bar{\sigma}(q)} \cdots \partial_{\bar{\sigma}(1)} \phi\left(\partial_{\bar{\sigma}(q+p)} \cdots \partial_{\bar{\sigma}(q+1)} \psi\left(b^{0}{ }_{<0>} \otimes \cdots \otimes b^{p+q-1}<0>b^{p+q}\right) \otimes\right. \\
\left.a^{0} \otimes b^{0}{ }_{<-p-q>} a^{1} \otimes \cdots \otimes b^{0}{ }_{<-1>} \cdots b^{p+q-1}{ }_{<-1>} a^{p+q}\right) .
\end{gathered}
$$

Similarly, by following [11], one uses cotraces on DG coalgebras to define a cup product that generalizes the characteristic map in Hopf cyclic cohomology. Indeed let $C$ be an $\mathcal{H}$-module coalgebra and $A$ be an $\mathcal{H}$-module algebra satisfying conditions (1)(4).

Proposition 5.3. Let $\phi \in C_{\mathcal{H}}^{q}(A, M)$ and $x:=m \otimes \otimes_{\mathcal{H}} c^{0} \otimes \cdots \otimes c^{p} \in C_{\mathcal{H}}^{p}(C, M)$ be Hopf cyclic cocycles. Then the following defines a cyclic cocycle in $C^{p+q}(A)$ :

$$
\begin{aligned}
& x \cup \phi\left(a_{0} \otimes a_{1} \otimes \cdots \otimes a_{p+q}\right)= \\
& \sum_{\sigma \in \operatorname{Sh}(q, p)}(-1)^{\sigma} \partial_{\bar{\sigma}(q)} \cdots \partial_{\bar{\sigma}(1)} \phi\left(\partial_{\bar{\sigma}(q+p)} \cdots \partial_{\bar{\sigma}(q+1)} x\left(a_{0} \otimes \cdots \otimes a_{p+q}\right)\right),
\end{aligned}
$$

where $\left(m \otimes c^{0} \otimes \cdots \otimes c^{n}\right)\left(a^{0} \otimes \cdots \otimes c^{n}\right):=m \otimes c^{0}\left(a_{0}\right) \otimes \cdots \otimes c^{n}\left(a_{n}\right)$.

Proof. The proof is similar to that of Proposition 5.2.

\section{References}

[1] A. Connes, Noncommutative differential geometry, Inst. Hautes Études Sci. Publ. Math. No. 62 (1985), 257-360.

[2] A. Connes and H. Moscovici, Background independent geometry and Hopf cyclic cohomology, arXiv:math.QA/0505475.

[3] A. Connes and H. Moscovici, Transgressions of the Godbillon-Vey class and Rademacher functions, in Noncommutative geometry and number theory, 79107, Aspects Math. E37, Vieweg, Wiesbaden, 2006.

[4] A. Connes and H. Moscovici, Hopf algebras, cyclic cohomology and the transverse index theorem, Comm. Math. Phys. 198 (1998), no. 1, 199-246. 
[5] M. Crainic, Cyclic cohomology of Hopf algebras, J. Pure Appl. Algebra 166 (2002), no. 1-2, 29-66.

[6] E. Getzler and J.D.S. Jones, The cyclic homology of crossed product algebras, J. reine angew. Math. 445 (1993), 163-174.

[7] A. Gorokhovsky, Secondary characteristic classes and cyclic cohomology of Hopf algebras, Topology 41 (2002), no. 5, 993-1016.

[8] P.M. Hajac, M. Khalkhali, B. Rangipour, and Y. Sommerhäuser, Stable antiYetter-Drinfeld modules, C. R. Math. Acad. Sci. Paris 338 (2004), no. 8, 587590.

[9] P.M. Hajac, M. Khalkhali, B. Rangipour, and Y. Sommerhäuser, Hopf-cyclic homology and cohomology with coefficients, C. R. Math. Acad. Sci. Paris 338 (2004), no. 9, 667-672.

[10] A. Kaygun, Bialgebra cyclic homology with coefficients, K-Theory 34 (2005), no. 2, 151-194.

[11] M. Khalkhali and B. Rangipour, Cup products in Hopf cyclic cohomology, C. R. Math. Acad. Sci. Paris 340 (2005), no. 1, 9-14.

[12] M. Khalkhali and B. Rangipour, Invariant cyclic homology, K-Theory 28 (2003), no. 2, 183-205.

[13] J.-L. Loday, Cyclic homology, Fundamental principles of mathematical sciences 301, Springer-Verlag, New York, 1992.

[14] H. Moscovici and B. Rangipour, Cyclic cohomology of Hopf algebras of transverse symmetries in codimension 1, Adv. Math. 210 (2007), no. 1, 323-374.

Bahram Rangipour bahram@unb.ca

Department of Mathematics and Statistics, University of New Brunswick, Fredericton, NB, E3B 5A3, Canada 